ARTICLE

\title{
Some Considerations in Devising Effective SCALE6/MAVRIC Models for Large Shielding Applications
}

\author{
Bojan PETROVIC* and David HARTMANGRUBER \\ Georgia Institute of Technology, Nuclear and Radiological Engineering, Atlanta 30332-0745, USA
}

\begin{abstract}
Analog Monte Carlo simulations for deep-penetration shielding problems are not viable; instead, aggressive use of variance reduction methods is used aiming to achieve acceptable accuracy for the result of interest in acceptable time. This requires developing effective variance reduction parameters, which in turn requires a systematic approach and an automated method. Frequently, a hybrid deterministic-stochastic methodology is deployed, where a deterministic transport theory method is used to generate adjoint function distribution. In the FW-CADIS method implemented in MAVRIC sequence of the SCALE6 package, deterministic forward calculation is performed as well, to enable generating variance reduction parameters for Monte Carlo simulation resulting in nearly-uniform statistical uncertainty over a large region. The choice of the deterministic model is user-controlled. However, the accuracy of the forward and adjoint deterministic solutions impacts the quality of Monte Carlo variance reduction parameters. We investigate this problem on a complex real-life shielding problem. The objective is to determine the radiation field (dose rate)

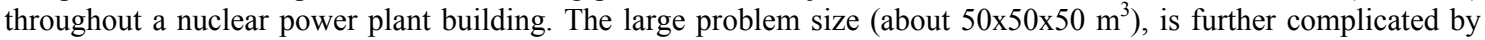
attenuation of more than 15 orders of magnitude. The results will serve as the initial step toward developing a general methodology for performance optimization of hybrid shielding calculations.
\end{abstract}

KEYWORDS: Monte Carlo shielding simulations, hybrid transport theory methods, automated variance reduction, MAVRIC sequence in SCALEG

\section{Introduction}

It has been recognized that deep-penetration shielding problems pose specific challenges to Monte Carlo simulations. Specifically, "straightforward" or analog simulations are not viable; instead, use of variance reduction methods is necessary to make analysis practical, i.e., achieve acceptable accuracy for the result of interest in acceptable time. This requires developing effective variance reduction parameters over a large phase space. Manually developing these parameters (based on trial and error) is in principle possible, but it requires significant engineering time and is not practical. Instead, theory-based approaches coupled to automated methods for generating variance reduction parameters have proved effective. In this paper, the FW-CADIS method implemented in MAVRIC sequence of the SCALE6 package is used. MAVRIC is based on a hybrid deterministic-stochastic methodology, where a deterministic transport theory method is used to generate adjoint function distribution. Additionally, deterministic forward calculation is performed to enable generating variance reduction parameters for Monte Carlo simulation resulting in nearly-uniform statistical uncertainty over a large region. The choice of the deterministic model is user-controlled. However, the accuracy of the forward and adjoint deterministic solutions impacts the quality of Monte Carlo variance reduction parameters.

We investigate this problem on a complex real-life shielding problem. IRIS is an advanced, smaller power PWR,

*Corresponding author, E-mail:Bojan.Petrovic@gatech.edu with aggressive dose reduction objectives. Due to its integral configuration, IRIS has a thick downcomer region which significantly reduces the radiation intensity outside the reactor vessel, forming the technical basis for achieving the objectives. However, this feature also makes the shielding analysis very challenging. The goal of evaluating the dose rate distribution throughout the IRIS nuclear power plant and, in particular, in all accessible areas further amplifies the problem. The large size of the problem and region of interest, about $50 \times 50 \times 50 \mathrm{~m}^{3}$, is further complicated by attenuation of more than 15 orders of magnitude.

The paper is organized as follows. Section II summarizes MAVRIC methodology. Section III describes the IRIS nuclear power plant and the MAVRIC model of IRIS. Section IV presents results and Section V provides conclusions.

\section{MAVRIC Methodology}

MAVRIC (MONACO with Automated Variance Reduction using Importance Calculations) is a new sequence within the SCALE6 code system for performing shielding calculations. ${ }^{1,2)}$ It uses a hybrid deterministic-stochastic method to generate variance reduction parameters for Monte Carlo simulations in an automated manner. MAVRIC is based on the CADIS (Consistent Adjoint Driven Importance Sampling) and FW-CADIS (Forward CADIS) methods. ${ }^{3-5)}$ Deterministic discrete ordinates code Denovo is used in forward and then in adjoint mode to obtain three dimensional flux and adjoint function distributions. MAVRIC uses this 
information to generate a space and energy dependent weight windows. In CADIS, the importance map biases the simulations to optimize obtaining results in a localized, phase-space area of interest. In FW-CADIS, the importance map biases the simulations in a way to obtain nearly-uniform statistical uncertainty throughout the region of interest. A consistent source biasing is used in both cases.

The FW-CADIS methodology and MONACO sequence may be summarized as follows. First the deterministically obtained scalar flux $\phi(r, E)$ is used to estimate the response of interest, $R(r, E)=\phi(r, E) \cdot f(r, E)$, where $f(r, E)$ is the response function, for example the detector (reaction) cross section $\sigma_{d}(E)$. This response is then used to construct the source for the adjoint problem

$$
q^{+}(r, E)=\frac{1}{R(r, E)} .
$$

Next, the deterministically obtained adjoint function is used to obtain the weight window targets that will be used in Monte Carlo simulations

$$
\bar{w}(r, E)=\frac{c}{\phi^{+}(r, E)},
$$

where $c$ is a normalizing constant. Additionally, the source distribution in Monte Carlo simulations will be biased consistently with weight windows, and the weight set to preserve the unbiased estimate. Additional details are available elsewhere. ${ }^{3-5)}$

In the described manner MAVRIC generates an importance map for Monte Carlo simulations that are performed using the Monaco code.

\section{IRIS Nuclear Power Plant}

\section{IRIS Nuclear Power Plant Layout}

IRIS is an advanced PWR with a nominal thermal output of 1,000 MWt and electric output of $\sim 335 \mathrm{MWe}$. It has an integral configuration with the primary circuit inside the reactor vessel. ${ }^{6,7)}$ The plan layout (containment and the reactor building arrangement) is shown in Fig. 1. The reactor building is $\sim 55$ meters tall, with the first 20 meters underground, and in its center the power plant contains a spherical containment vessel that is 25 meters in diameter. The pressure vessel (Fig. 2) is 3.39 meters in outer radius and 22.27 meters tall. Due to the integral design, the downcomer region is 1.67 meters wide, i.e., around 8 times thicker than in a typical PWR design, which significantly reduces the radiation dose rate outside the reactor pressure vessel. At the same time, the shielding analysis to determine the dose rate outside the vessel becomes significantly more difficult, providing a well suited problem to examine the issues of effective simulations using the MAVRIC methodology.

IRIS aims is to notably reduce the collective dose as compared with the current nuclear power plants. ${ }^{8)}$ Consequently, the goal is to have a dose rate to personnel in accessible areas lower than $0.25 \mu \mathrm{Sv} / \mathrm{hr}$. Because of the compact IRIS design and proximity of accessible areas to the

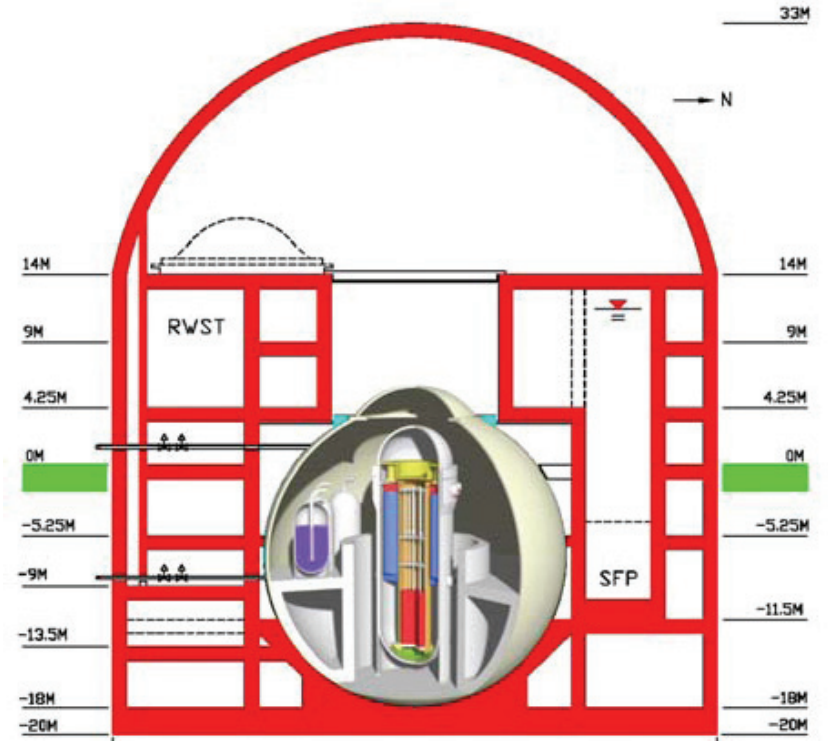

Fig. 1 IRIS reactor building with containment and pressure vessel shown

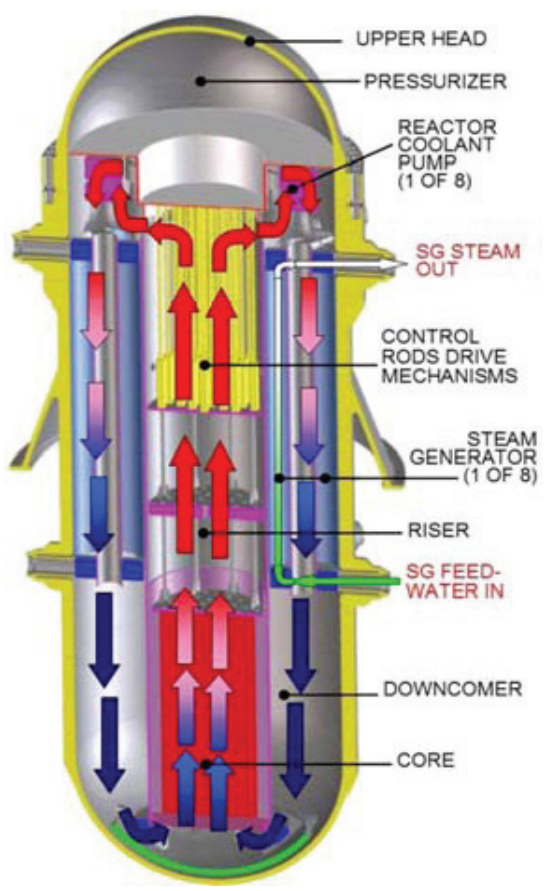

Fig. 2 IRIS integral reactor vessel layout

reactor vessel and the presence of potential steaming paths, accurate modeling and simulations are required to obtain reliable results.

\section{MAVRIC Model of IRIS}

MAVRIC model of the IRIS vessel and reactor building has been developed to accurately represent the vessel, containment and concrete structures. Figures 3 to $\mathbf{5}$ depict the $3 \mathrm{D}$ view, and vertical and radial cross sectional cuts of the IRIS nuclear power plant model with grids depicting dimensions of the model in meters, through the center and at ground level elevation, respectively. 


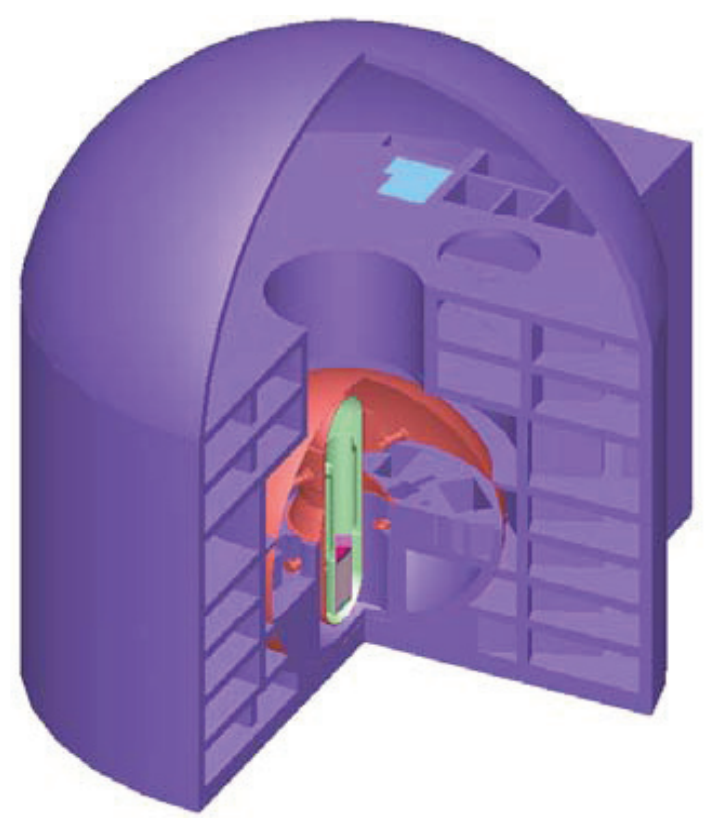

Fig. 3 IRIS model of IRIS - 3D perspective view

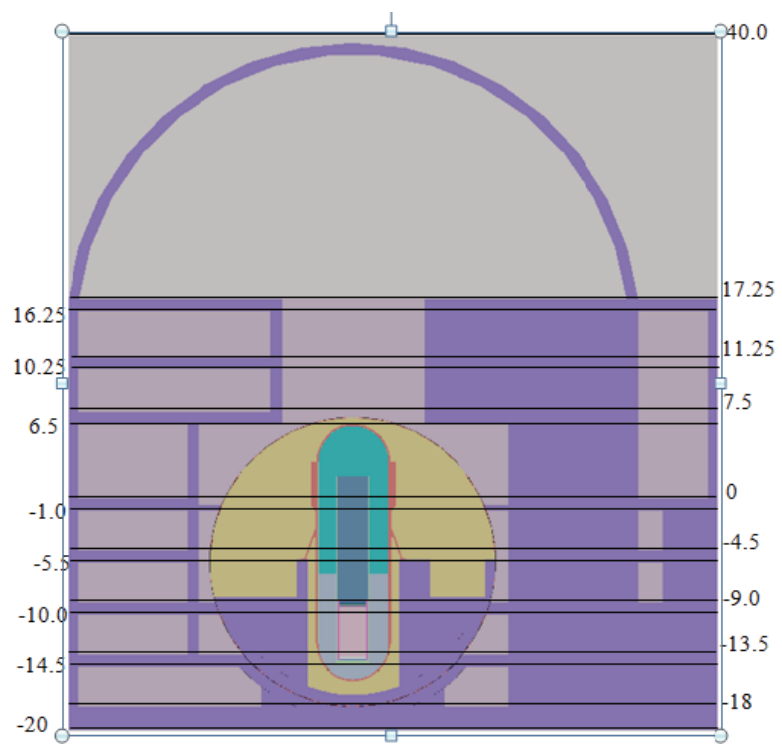

Fig. 4 IRIS model - vertical cross section

Focus of this study was the dose rate determination in the control room, marked by "CR" in Figs. 2 and 3. The control room is located underground, extending between the elevations of $-4.5 \mathrm{~m}$ and $-1.0 \mathrm{~m}$.

\section{MAVRIC Simulations and Analysis of Results}

To determine dose rate in the control room and between the reactor vessel and control room, MAVRIC model as shown in Fig. 6 was devised. The importance map (the blue encompassing region) denotes the region that will be considered in the model and for which the variance reduction parameters will be generated. The forward source area corresponds to the fission source, i.e., reactor core. The adjoint source area indicates the region for which the methodology

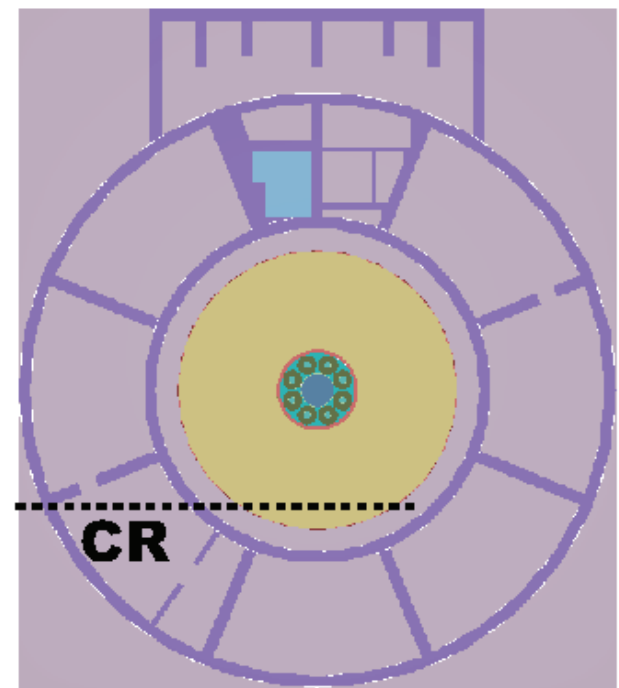

Fig. 5 IRIS model - radial cross section at elevation within the control room ( $-4.5 \mathrm{~m}$ to $-1.0 \mathrm{~m}$ below the ground)

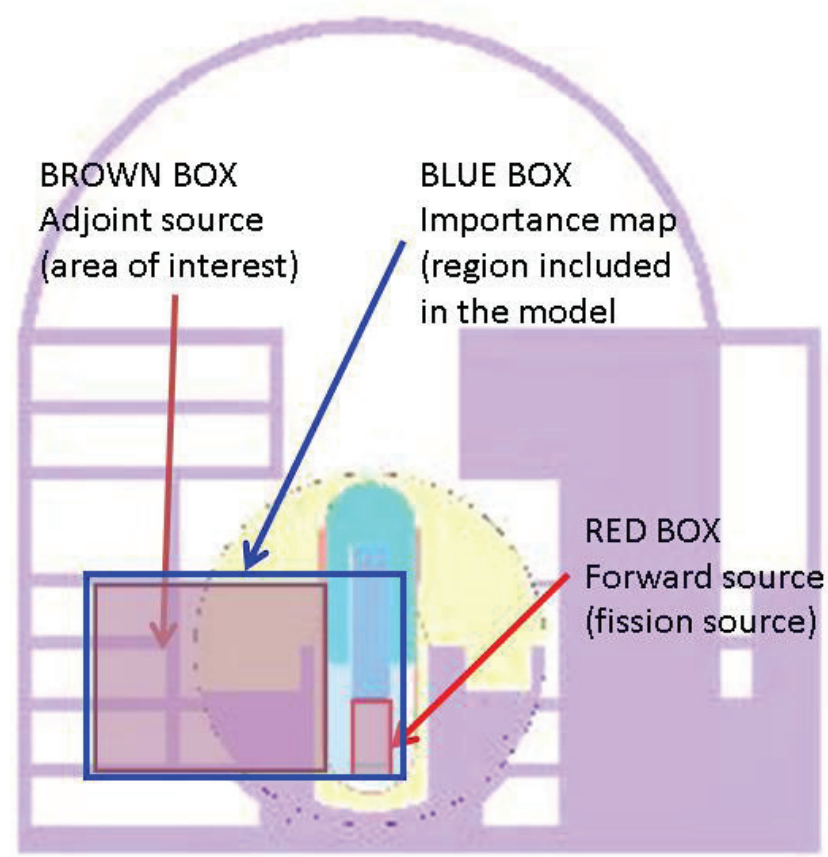

Fig. 6 IRIS model - radial cross section at control room level elevation ( $-4.5 \mathrm{~m}$ to $-1 \mathrm{~m}$ below ground)

will aim to optimize generation of Monte Carlo results, i.e., minimize statistical uncertainty while producing results with spatially near-uniform statistical uncertainty. While we are primarily interested in the radiation dose in the control room, in this starting analysis we aim to generate results on a broader region, i.e., also in the area between the core and control room, to confirm physically expected behavior of results and thus increase confidence in results.

The region covered by this model is $20.86 \times 20.86$ $\times 15.76 \mathrm{~m}^{3}$ large. In deterministic Denovo calculations it was covered by 200 intervals in each direction, i.e., a total of 8 million meshes. $\mathrm{P}_{1} \mathrm{~S}_{4}$ approximation was used. Calculations were performed on a Unix machine (utilizing a single 


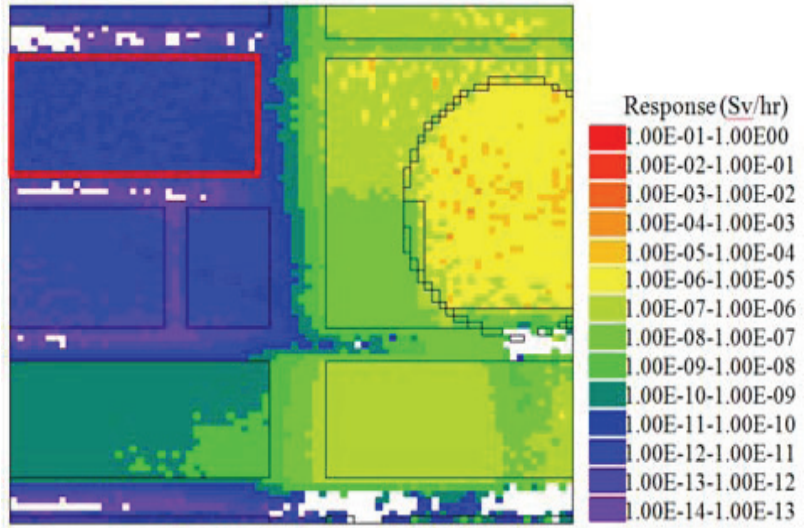

Fig. 7 Radial plane cut of the dose rate distribution at 12 meters south from the center of the containment vessel

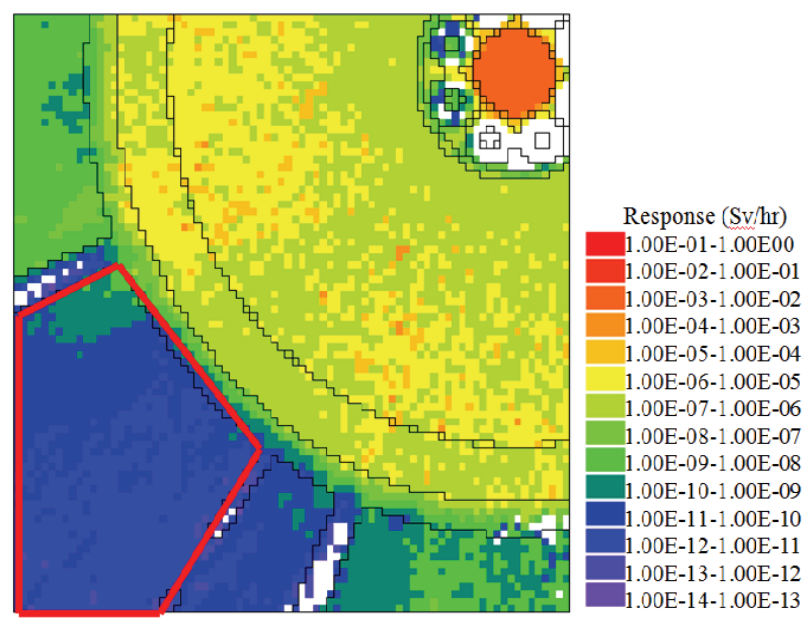

Fig. 8 Radial plane cut of the dose rate distribution at an elevation through the control room (1.05 $\mathrm{m}$ below the ground level)

CPU).

The mesh tally for Mont Carlo simulations was set to $80 \times 80 \times 80$ meshes. This spatial resolution of tallies was deemed appropriate for the purpose of this study.

A dose rate distribution in the vertical plane (indicated by the dashed line in Fig. 5) which is 12 meters "south" from the core center, and in the radial plane at elevation of $-1.05 \mathrm{~m}$, are shown in Figs. 7 and 8. The control room is indicated by the red box.

This simulation had 10 batches with 1 million particles each. The total run time for the calculations was 50.44 hours. The Denovo portion of the code took 2.79 hours for the forward part of the calculations and the adjoint portion of the program took 2.61 hours. The Monaco portion of the program sequence took 42.58 hours. From the results it can be estimated that the dose rate in the control room has a maximum around $10^{-9} \mathrm{~Sv} / \mathrm{hr}\left(10^{-7} \mathrm{rem} / \mathrm{hr}\right)$, meeting the IRIS dose reduction objectives. These results, obtained with a moderate running time, enabled getting a useful estimate of the dose rate in the relatively large considered region of the building. The statistical uncertainty in Monaco results is relatively high, which can be seen in Fig. 9, but still adequate for a scoping study aimed to determining the dose rate distribution over a

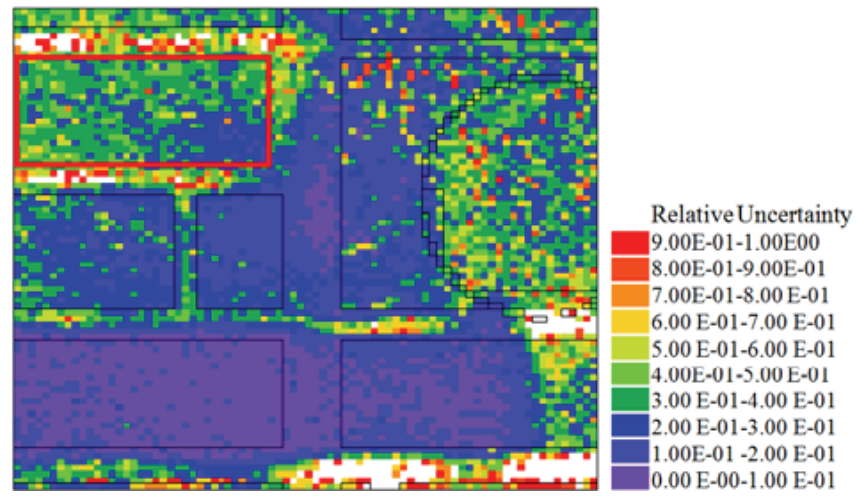

Fig. 9 Vertical plane cut of the relative uncertainty distribution at 12 meters south from the center of the containment vessel

large volume.

From Fig. 9 it may be further observed that the relative uncertainty ranges from 0.1 to 0.6 with a representative value of 0.4 in the control room. Given the size of the problem and attenuation by many orders of magnitude, this is an impressive result. The uncertainty distribution, while not fully uniform, is much more uniform than what could have been achieved by any manual trial-end-error variance reduction. Moreover, it was obtained in an automated manner with limited use of engineering time and the model may be easily modified to generate results in other sections of the plant. Note that the targeted uniform-statistics area denoted by the blue box in Fig. 6 does not include the rightmost side of Fig. 9 area; the poor statistics there is induced by the user-selected problem definition, and appropriate for the objective.

A closer look at the non-uniform uncertainty distribution reveals the deterministic mesh as the main culprit. This initial analysis was performed using spatial meshes that are both quite large, and spatially uniform. The latter means that they do not account for the characteristics of this model, which has alternating layers of highly absorbing and very transparent materials (concrete and air).

Using a finer and/or tailored non-uniform mesh is expected to further improve the uniformity of the statistical uncertainty. Analysis is in progress and additional results are being obtained to quantify the level of improvement and the required additional computational efforts. One example is provided in Fig. 10, showing the distribution of statistical uncertainties for an improved simulation model. The simulation was optimized to generate results in all potentially accessible areas (i.e., "rooms" on the left side and bottom of the figure). A more uniform distribution is achieved, with relative uncertainties generally in the range below 0.3 in the areas of interest.

Finally, a simulation focusing on control room only provided results with uncertainties shown in Fig. 11. The Monte Carlo portion of simulations in this analysis used 3 to 4 times less CPU time than the previous simulations, yet provided better statistics in the control room. This is of course expected since the region of interest is smaller. However, by itself, with the surrounding areas blank (i.e., without any results generated) it could raise questions about the validity of results. 


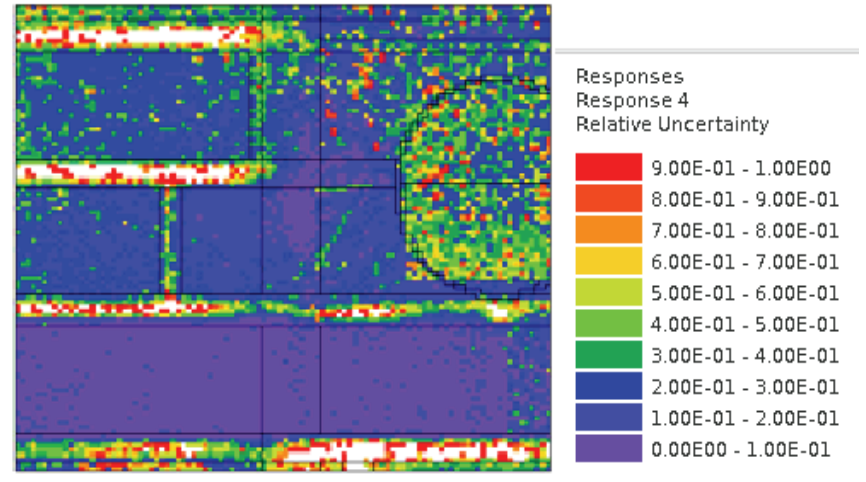

Fig. 10 Vertical plane cut of the relative uncertainty distribution at 12 meters south from the center of the containment vessel obtained with improved variance reduction parameters

Having the ability in MAVRIC to easily obtain both the global solution (Figs. 7-9) and more focused solution(s) enables both verifying the overall physical behavior and reducing the statistical uncertainty in the primary area of interest.

\section{Conclusion}

A challenging shielding analysis was performed using the SCALE6/MAVRIC sequence. The dose rate distribution was obtained over a large volume, in moderate time, and with an acceptable accuracy for the purpose of the scoping study. A more focused solution (dose distribution in the control room) but with improved statistics was obtained as well.

The automated MAVRIC sequence facilitates obtaining multiple solutions effectively, both from the standpoint of simulations efficiency (variance reduction) and of minimizing the engineering efforts. Nevertheless, large challenging problems, such as the one analyzed, still require judicious use of available computational resources and understanding of the underlying physics; further studies are underway to assess approaches to optimizing the overall performance of this hybrid deterministic-stochastic methodology.

\section{References}

1) SCALE: A Modular Code System for Performing Standardized Computer Analyses for Licensing Evaluation, ORNL/TM-2005/39, Version 6, Vols. I-III (2009), available

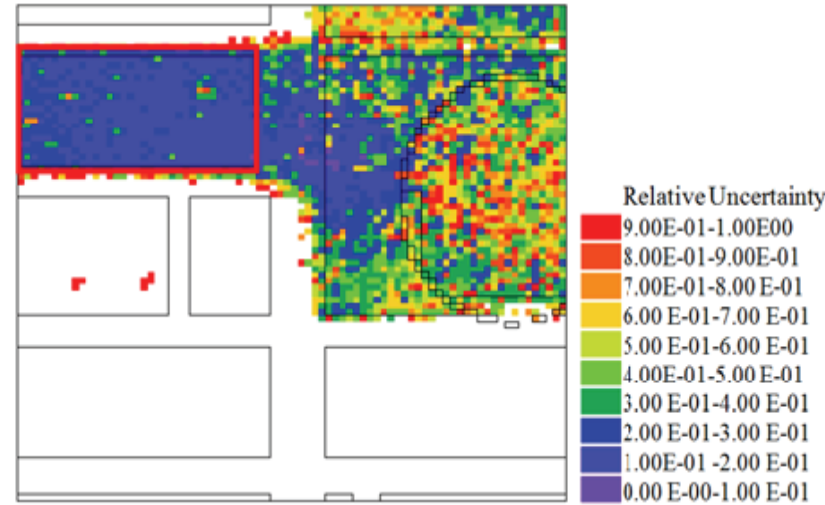

Fig. 11 Vertical plane cut of the relative uncertainty distribution at 12 meters south from the center of the containment vessel obtained with region of interest specified as control room only

from Radiation Safety Information Computational Center at Oak Ridge National Laboratory as C00750.

2) D. E. Peplow, MAVRIC: MONACO with Automated Variance Reduction using Importance Calculations, ORNL/TM-2005/39, Version 6, Vol. I, Sect. S6, Oak Ridge National Laboratory (ORNL) (2009).

3) J. C. Wagner, A. Haghighat, "Automated Variance Reduction of Monte Carlo Shielding Calculations Using the Discrete Ordinates Adjoint Function,” Nucl. Sci. Eng., 128, 186 (1998).

4) J. C. Wagner, E. D. Blakeman, D. E. Peplow, "Forward-Weighted CADIS Method for Global Variance Reduction," Trans. Am. Nucl. Soc., 97, 630-633 (2007).

5) D. E. Peplow, T. M. Evans, J. C. Wagner, "Simultaneous Optimization of Tallies in Difficult Shielding Problems," Nucl. Technol., 168, 785-792 (2009).

6) M. D. Carelli, L. E. Conway, L. Oriani, B. Petrovic, C. V. Lombardi, M. E. Ricotti, A.C.O. Barroso, J. M. Collado, L. Cinotti, N. E. Todreas, D. Grgic, M. M. Moraes, R. D. Boroughs, H. Ninokata, D. T. Ingersoll, F. Oriolo, "The Design and Safety Features of the IRIS Reactor," Nucl. Eng. Des., 230, 151-167 (2004).

7) M. Carelli, B. Petrovic, "Here's Looking at IRIS," Nucl. Eng. Int., 51[620], 12-18 (2006).

8) B. Petrovic et al., "Design Features of the IRIS Reactor to Reduce Collective Dose During Operation and Maintenance, and to Simplify D\&D," Proc. 8th Intl. Conf. on Nuclear Option in Countries with Small and Medium Electricity Grids, Dubrovnik, Croatia, May 16-20, 2010; HND (2010). 